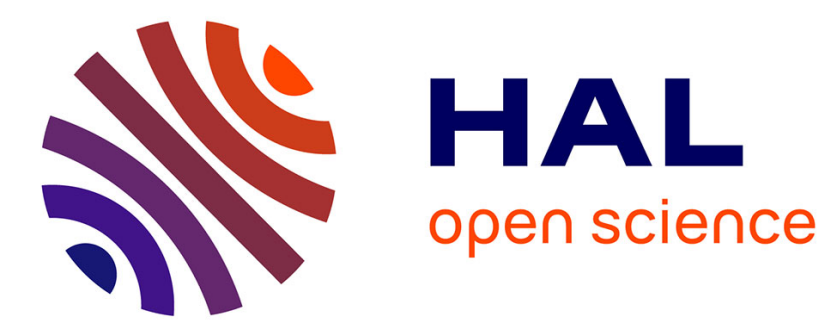

\title{
Dispositifs cryogéniques pour les applications de la supraconductivité
}

\author{
A. Lacaze
}

\section{To cite this version:}

A. Lacaze. Dispositifs cryogéniques pour les applications de la supraconductivité. Revue de Physique Appliquée, 1969, 4 (4), pp.589-591. 10.1051/rphysap:0196900404058900 . jpa-00243339

\section{HAL Id: jpa-00243339 https://hal.science/jpa-00243339}

Submitted on 1 Jan 1969

HAL is a multi-disciplinary open access archive for the deposit and dissemination of scientific research documents, whether they are published or not. The documents may come from teaching and research institutions in France or abroad, or from public or private research centers.
L'archive ouverte pluridisciplinaire HAL, est destinée au dépôt et à la diffusion de documents scientifiques de niveau recherche, publiés ou non, émanant des établissements d'enseignement et de recherche français ou étrangers, des laboratoires publics ou privés. 


\title{
DISPOSITIFS GRYOGÉNIQUES POUR LES APPLIGATIONS DE LA SUPRAGONDUGTIVITÉ
}

\author{
A. LACAZE.
}

Cette table ronde qui a réuni 40 participants avait pour but de faire le tour d'horizon des questions techniques posées par l'utilisation des très basses températures dans le domaine de la supraconductivité, et de dégager l'effort français dans ce domaine. Les points généraux étudiés sont : la production, le transfert et le stockage de l'hélium liquide, l'isolation thermique et le transfert de chaleur, la mesure et la régulation de température dans le domaine de 1 à $15^{\circ} \mathrm{K}$.

Tous les participants étant intervenus dans une discussion très animée, nous donnerons seulement un aperçu d'ensemble de la réunion.

1. Production des basses températures. - On peut refroidir un système quelconque, dans ce domaine de température, par deux voies différentes :

- vaporisation d'hélium liquide provenant d'un gros liquéfacteur extérieur,

- coupler le système à un réfrigérateur continu.

La première méthode est presque toujours utilisée dans les laboratoires où les quantités de chaleur à enlever ne sont pas trop grandes et où le fonctionnement ne dure qu'un temps court. La baisse du prix de l'hélium liquide qui est actuellement de l'ordre de $35 \mathrm{~F}$ le litre, gaz compris, a beaucoup contribué à développer cette technique. Il est cependant fait remarquer qu'il est bon de ne pas rejeter l'hélium vaporisé dans les salles de mesure si l'on ne veut pas perturber le fonctionnement des détecteurs de fuite à hélium.

Dans cette méthode, la plus grande partie de la chaleur sensible du gaz évaporé est perdue. Le rendement de ce procédé est donc faible. Thermodynamiquement, pour enlever $1 \mathrm{~W}$ au point normal d'ébullition de l'hélium, il faut dépenser une puissance à 300 oK de $326 \mathrm{~W}$.

Dans la seconde méthode, les vapeurs formées à 4,2 oK sont immédiatement récupérées dans le réfrigérateur et la puissance à fournir à 300 oK n'est, théoriquement, que de $70 \mathrm{~W}$. On a donc un rendement près de 5 fois plus grand et on a tout intérêt, pour les installations importantes, fonctionnant de manière continue, à incorporer un réfrigérateur au système.

Les liquéfacteurs, ou les réfrigérateurs dans ce domaine de température, utilisent toujours la détente de Joule-Thomson de l'hélium pour l'étage à plus basse température. L'hélium doit être d'abord prérefroidi en dessous de sa température d'inversion de l'effet Joule-Thomson; ceci peut être obtenu soit par vaporisation d'azote liquide suivi d'une détente de Joule-Thomson de l'hydrogène, soit par détente dans une machine de l'hélium. Le premier procédé a été utilisé sur les premiers liquéfacteurs à cause de sa plus grande fiabilité. Avec les progrès de la technique, le second procédé se généralise de plus en plus.

Dans les installations de petite puissance, les détendeurs à pistons sont utilisés dans des cycles avec échangeurs à contre-courant ou avec récupérateurs de chaleur. Pour les grosses installations, les cycles avec turbine et échangeur sont adoptés de manière générale. Les turbines de détente à hélium doivent tourner à très grande vitesse et sont souvent à palier à gaz. Il semble que la limite inférieure d'utilisation des turbines dans les réfrigérateurs à hélium se situe vers $100 \mathrm{~W}$ à $4,2 \mathrm{o}$, à cause de la perte de rendement qu'entraînerait une miniaturisation plus poussée des turbines.

En pratique, le rendement total des installations n'atteint que $10 \%$ du rendement de Carnot pour des puissances de réfrigération comprises entre 10 et $100 \mathrm{~W}$ à $4,2 \mathrm{oK}$, que $1 \%$ pour des puissances 100 fois plus faibles. Même pour des installations 100 fois plus grosses, on n'atteint pas $20 \%$ du rendement de Carnot. Une limitation importante de rendement provient des compresseurs et il devrait être possible dans l'avenir d'améliorer considérablement ce facteur. A la question de savoir s'il serait plus intéressant d'effectuer la recompression du gaz à basse température au lieu de le ramener à l'ambiante, on peut répondre négativement. En effet, si, théoriquement, le résultat serait le même, dans la réalité, du fait des pertes des compresseurs, pertes qu'il faudrait enlever par exemple avec de l'azote liquide, le rendement de l'ensemble serait plus mauvais. Il faut noter que le problème serait différent pour un réfrigérateur placé dans l'espace interstellaire. 
La réfrigération dans cette région de température commence seulement à entrer dans le domaine courant, et il est difficile de donner un prix d'installation. D'ailleurs, la plupart des installations sont construites pour une utilisation particulière. Cependant, il semble qu'un réfrigérateur de $100 \mathrm{~W}$ à $4,2^{\circ} \mathrm{K}$ coûte environ $700000 \mathrm{~F}$. Ce prix pourrait changer notablement si le marché se développait. La température de $4,2 \circ \mathrm{K}$ a été choisie à cause du point d'ébullition normal de l'hélium, mais si on pouvait utiliser les supraconducteurs à $8 \mathrm{oK}$, on doublerait presque le rendement.

Sur le plan international, on trouve réalisées, ou en projet, toute les tailles de réfrigérateurs de $1 / 2 \mathrm{~W}$ à $5 \mathrm{~kW}$ à $4,2 \circ \mathrm{K}$. Parmi les réalisations, on peut noter un liquéfacteur de 800 1/h (Sulzer, Otis, Kansas), deux réfrigérateurs de $300 \mathrm{~W}$ à $1,8{ }^{\circ} \mathrm{K}$ ( $\mathrm{ADL}$ à Stanford et Linde à Karlsruhe). Sur le plan français, en dehors des installations à détente de Joule-Thomson, on a la mise au point d'un réfrigérateur à piston de l'ordre du watt (Alcatel) et des turbines pour réfrigérateurs de 100 à $1000 \mathrm{~W}$ (Air Liquide). Il semble que peu de personnes soient actuellement intéressées par la réfrigération en dessous de $4 \mathrm{oK}$.

Une mention est faite des réfrigérateurs à dilution de ${ }^{3} \mathrm{He}$ dans ${ }^{4} \mathrm{He}$, qui actuellement descendent en dessous de $30 \mathrm{~m}$ oK (Orsay et Grenoble) et au moins 5 laboratoires seraient intéressés, en France, par ces appareils dans les 2 ans.

2. Transfert du froid. - Avec les installations de réfrigération continue, se pose le problème du transfert du froid de la production à l'utilisation.

En général, la réfrigération est effectuée par transfert d'hélium liquide à travers des pipe-lines de petite longueur. L'avantage du liquide est de fournir un bon coefficient d'échange entre paroi métallique et fluide, et de donner une réserve de frigories en cas de panne de l'installation.

Dans certains cas cependant, il peut être avantageux d'utiliser du gaz froid sous haute pression pour éliminer les problèmes de l'écoulement en double phase et des blocages de conduite qui peuvent en résulter. Ce mode de réfrigération peut permettre, en plus, d'effectuer la réfrigération à plus haute température, donc avec un meilleur rendement. Les échanges dans le gaz étant moins bons, il est nécessaire d'utiliser l'hélium en convection forcée avec de grandes vitesses de circulation. Il est alors possible d'obtenir des coefficients de convection de quelques dixièmes de watt par $\mathrm{cm}^{2} \mathrm{oK}$.

On a tout intérêt à placer le réfrigérateur le plus près possible de l'objet à refroidir. Cependant, si plusieurs points doivent être refroidis, il est plus économique de prendre un seul réfrigérateur de meilleur rendement et des lignes de transfert. Typiquement, pour une ligne devant enlever $1 \mathrm{~kW}$ à $100 \mathrm{~m}$, avec une conduite sous vide, les pertes seraient de l'ordre de $100 \mathrm{~W}$; avec un écran à température de l'azote liquide, ou du superisolant, ces pertes pourraient être ramenées à $1 \mathrm{~W}$ et seraient alors négligeables. Il ne semble cependant pas qu'il y ait actuellement d'expérience de ligne de transfert à grande distance.

Pour la réfrigération se pose le problème de la circulation du fluide. Il semble que la solution la plus simple consiste à utiliser directement la haute pression disponible dans le réfrigérateur. Il existe des pompes pour oxygène ou hydrogène liquide; mais pas à notre connaissance, pour l'hélium liquide ou supercritique. D'ailleurs, la plupart de ces pompes ne fonctionnent que pendant des temps courts. Il est signalé que des moteurs fonctionnant à basses températures ont été mis au point en France :

a) Pour gyroscope tournant à $900 \mathrm{t} / \mathrm{s}$ (Alcatel);

b) Avec arbre en acier sur coussinet en saphir (O.N.E.R.A.);

c) A effet Hall (L.C.I.E.), mais il est probable que tous ces moteurs ont des pertes prohibitives. Le problème du circulateur fiable à basse température reste donc à résoudre.

3. Vases de stockage et cryostats. - Actuellement, pour les vases de stockage, le seul isolement intéressant est le superisolant (feuilles minces d'aluminium séparées par des voiles isolants) avec interposition de feuilles de cuivre refroidies par les vapeurs, le tout sous vide moléculaire. Les vases de $100 \mathrm{l}$ et bientôt 501 français sont parfaitement compétitifs.

Pour les cryostats, la chemise à azote liquide n'est intéressante que si elle est utilisée pour prérefroidir de gros dispositifs internes avant injection de l'hélium. Elle est d'autre part intéressante pour des appareils fonctionnant pendant des temps courts pour lesquels le superisolant n'aurait pas le temps de se mettre en équilibre. Dans ces conditions cependant, le cryostat comportant un écran refroidi par les vapeurs ou le cryostat à circulation est plus économique. Lorsque la forme du cryostat est simple, on peut utiliser du mylar aluminisé, maintenant commercialisé, qui, bien que le plus cher en poids, est un des moins chers à égalité de puissance calorifique transmise.

La mise en œuvre du superisolant est cependant délicate et il est noté que parfois le prix du vase superisolé est supérieur à celui du vase avec écran d'azote liquide.

Une discussion suit sur les intérêts comparés des cryostats métalliques et des cryostats en verre. Il est noté que l'hélium diffuse à travers le pyrex, cette diffusion étant d'ailleurs d'autant plus grande que la température est plus élevée. La possibilité actuelle de réaliser des fenêtres optiques en matériaux les plus divers fait perdre beaucoup d'intérêt au cryostat tout en verre. 
4. Mesure et régulation de température. - La définition de la température de 0,1 à $10 \mathrm{oK}$ à $10^{-2} \mathrm{oK}$ est très difficile. Par contre, une stabilisation à $10^{-3}{ }^{\circ} \mathrm{K}$ est relativement facile à obtenir, des stabilisations à $10^{-5}$ et même $10^{-6} \mathrm{o} \mathrm{K}$ sont citées.

Par régulation du débit de pompage sur un bain, il est difficile d'obtenir une bonne stabilisation de la température, car une remontée de pression ne veut pas dire une remontée de température, sauf avec l'hélium II. La meilleure solution consiste à régler grossièrement le débit de pompage, puis à stabiliser la température par chauffage du bain ou de l'objet. Le repérage de la température se fait au moyen de résistance au carbone ou de sondes au germanium dopé. Pour de petites surfaces, il est possible d'utiliser du graphite colloïdal.
Conclusions. - Il est souhaité de manière générale qu'une standardisation du matériel cryogénique soit adoptée, mais ceci ne peut se faire que par accord entre tous les constructeurs au moins sur le plan européen.

Il est constaté que le principal problème en cryogénie est celui du manque d'informations entre laboratoires. Sur ce point, la proposition de diffusion d'un travail de compilation des propriétés des matériaux à B.T. fait à l'O.N.E.R.A. est accueillie très favorablement. Tous les membres de cette table ronde demandent qu'une personne accepte de lancer un bulletin de liaison genre "Cryophysics Newsletter » qui éviterait qu'un chercheur isolé perde du temps à mettre au point ce qui existe dans un laboratoire voisin. 